\title{
Joint Distributed Source-Channel Decoding for LDPC-Coded Binary Markov Sources
}

\author{
Reza Asvadi*, Tad Matsumoto*†, and Markku Juntti* \\ * Center for Wireless Communications (CWC), University of Oulu, Finland \\ Emails: \{rasvadi, tadashi.matsumoto, markku.juntti\}@ee.oulu.fi \\ $\dagger$ Japan Advanced Institute of Science and Technology, 1-1 Asahi-Dai, Nomi, Ishikawa, Japan \\ Email: matumoto@jaist.ac.jp
}

\begin{abstract}
We propose a novel joint decoding technique for distributed source-channel (DSC) coded systems for transmission of correlated binary Markov sources over additive white Gaussian noise (AWGN) channels. In the proposed scheme, relatively shortlength, low-density parity-check (LDPC) codes are independently used to encode the bit sequences of each source. To reconstruct the original bit sequence, a joint source-channel decoding (JSCD) technique is proposed which exploits the knowledge of both temporal and source correlations. The JSCD technique is composed of two stages, which are iteratively performed. First, a sum-product (SP) decoder is serially concatenated with a BCJR decoder, where the knowledge of source memory is utilized during local (horizontal) iterations. Then, the estimate of correlation between the sources is used to update the concatenated decoder during global (vertical) iterations. Therefore, the correlation of the sources is assumed as side information in the subsequent global iteration of each concatenated decoder. From the simulation results of frame/bit error rate (FER/BER), we note that significant gains are achieved by the proposed decoding scheme with respect to the case where the correlation knowledge is not completely utilized at the decoder.
\end{abstract}

\section{INTRODUCTION}

In distributed source coding problem, Slepian-Wolf $(S W)$ theorem states that using joint decoding achieves higher compression rates compared to separate decoding of correlated sources [1]. Numerous distributed compression methods have been developed for non-uniform sources or sources with memory during recent years in the context of sensor networks, e.g., [2], [3], and references therein.

Although there are many efficient coding methods for lossless compression, transmission of correlated sources over noisy channels based on the SW cooperation has been investigated quite recently [4]-[7]. To exploit the benefit of the SW cooperation over the relay channels, joint source-channel decoding (JSCD) techniques have been recently proposed based on turbo coding with significant bit error rate (BER) performance gain [8], [9]. Turbo-like codes which are used for encoding of correlated sources are assumed, having long block-lengths and hence the decoding latency is a problem. Thus, these codes are not suitable in certain practical applications due to decoding latency and/or power consumption limitations.

Furthermore, most of the research results on transmission of correlated bit sequences over noisy channels focus on sources without temporal correlation (source memory), and hence the sources are assumed to be independent and identically distributed (i.i.d) in the time domain. In [5] and [6], turbo and low-density generator-matrix (LDGM) codes were proposed for transmission of correlated binary i.i.d sources over additive white Gaussian noise (AWGN) channel, respectively. Also in [7], a joint iterative decoder of LDPC codes was introduced for correlated sources without memory. The decoding technique proposed in [7] employs a two-stage iterative decoder, between which local and global iterations are performed. In the local iterations, decoding is performed using the sum-product (SP) algorithm [10], while in the global iterations the estimate of correlation between the sources is passed on to the SP decoder to improve the decoding performance in the subsequent round of local iterations.

The main objective of this paper is to propose a novel technique for the transmission of two correlated binary sequences following a first order Markov process, in contrast to [5][7] where the sources are i.i.d. Relatively short-length (length $<$ 10000) LDPC codes with systematic parity-check matrices are used for the encoding of bit sequences to be transmitted because efficient performance improvement can be expected with the help of the correlation within and across the sequences in both the waterfall and error floor regions.

At the decoder side, the joint decoder proposed in this paper exploits the information related to the temporal and source correlations in two stages. A standard BCJR decoder [11] is serially concatenated to a SP decoder for the efficient utilization of the temporal memory structure inherent within the output sequences of the source. Additionally, to exploit the source correlation, the method proposed in [7] is used, where the log-likelihood ratio $(L L R)$ of the local iterations is updated according to the knowledge about the source correlation estimated in the previous global iteration. Numerical results demonstrate that significant BER performance gains are achieved by the proposed JSCD method for the correlated binary sequences generated by a Markov source.

The organization of the paper is as follows. In Section $\amalg$ basic concepts and notations related to the proposed algorithm are introduced. In Section IIII the proposed joint decoder structure is described in details. In Section [IV] simulation results and comparisons with decoders that do not exploit the temporal and source correlations are provided. Finally, Section $\nabla$ concludes the paper. 


\section{PRELIMINARIES}

\section{A. Source Model}

We consider two binary sources, one of them is modeled as a binary Markov source whose sequences are denoted by $\mathbf{b}^{1}=$ $\left\{b_{1}^{1}, b_{2}^{1}, \ldots\right\}$, and the sequences of another source, denoted by $\mathbf{b}^{2}=\left\{b_{1}^{2}, b_{2}^{2}, \ldots\right\}$, is assumed to be erroneous version of $\mathbf{b}^{1}$, corrupted by a binary noise with occurrence probability $p$, where $b_{i}^{q} \in\{0,1\}, q=1,2$. Therefore, $b_{i}^{2}=b_{i}^{1} \oplus z_{i}$, where $Z_{i}$ and $\oplus$ denote a binary random variable with probability $p_{r}\left(z_{i}=1\right)=p$ and a modulo- 2 addition, respectively. The probability $1-p$ determines correlation ratio between the bit sequences of the two sources, i.e., the smaller the $p$ is, the more correlated the sources are.

The binary Markov source considered in this paper is a stationary, ergodic, state emitting Markov source $\left\{S_{t}\right\}$, whose transition probabilities are described by the transition matrix

$$
\Pi=\left[\pi_{i j}\right]=\left[\begin{array}{cc}
1-\alpha & \alpha \\
\beta & 1-\beta
\end{array}\right],
$$

where the transition probability is defined

$$
\pi_{i j} \triangleq p_{r}\left\{S_{t}=j \mid S_{t-1}=i\right\}, \quad i, j \in\{0,1\} .
$$

The stationary distribution of the source states is denoted by $\left(\mu_{0}, \mu_{1}\right)$ where $\mu_{0} \triangleq p_{r}\left\{S_{t}=0\right\} \triangleq 1-\mu_{1}$. With the stationarity assumption, it can be easily shown that $\mu_{0}$ and $\mu_{1}$ are $\mu_{0}=1-\mu_{1}=\frac{\beta}{\alpha+\beta}$. If $\alpha \neq \beta$, the source described above is an asymmetric binary Markov source. Otherwise, when $\alpha=$ $\beta \neq 1 / 2$ the source is a symmetric Markov source with a uniform stationary distribution, i.e., $\mu_{0}=\mu_{1}=1 / 2$.

The entropy rate of stationary binary Markov source is given by

$$
H(s)=\mu_{0} h(\alpha)+\mu_{1} h(\beta),
$$

where $h(p)=-p \log _{2}(p)-(1-p) \log _{2}(1-p)$ is the binary entropy function [12].

We will assume in this paper that the Markov source characteristics, i.e., $\alpha$ and $\beta$, are known to the proposed decoder. Estimation of Markov source parameters was discussed for unknown temporal correlation parameters in [13].

\section{B. LDPC Codes}

Consider a binary LDPC code $\mathcal{C}$ represented by a Tanner graph $G=\left(V_{b} \cup V_{c}, E\right)$, where $V_{b}=\left\{v_{1}, \ldots, v_{n}\right\}$ and $V_{c}=$ $\left\{c_{1}, \ldots, c_{m}\right\}$ are the sets of variable nodes and check nodes, respectively, and $E$ is the set of edges. Corresponding to $G$, we have an $m \times n$ parity-check matrix $H=\left[h_{i j}\right]$ of $\mathcal{C}$, where $h_{i j}=1$ if and only if (iff) the node $c_{i} \in V_{c}$ is connected to the node $v_{j} \in V_{b}$ in $G$; or equivalently, iff $\left\{v_{j}, c_{i}\right\} \in E$. The code rate of $\mathcal{C}$ is $R_{c}=1-\frac{\operatorname{rank}(H)}{n}$, where $\operatorname{rank}($.$) is the rank$ of its argument matrix in $G F(2)$.

The degree of a variable or a check node is the number of nodes connected to it. If the nodes in the set $V_{b}$ and/or the nodes in the set $V_{c}$ have different degrees, the corresponding LDPC code is called irregular. For an irregular LDPC code, the degree distribution of variable nodes is described by the polynomial, $\lambda(x)=\sum_{i=2}^{D_{v}} \lambda_{i} x^{i-1}$, where $D_{v}$ is the maximum variable node degree and $\lambda_{i}$ is the fraction of the edges connected to the variable nodes of degree $i$.

\section{PROPOSED SYSTEM MODEL}

\section{A. LDPC Encoder}

In our proposed scheme, two individual LDPC codes are used for encoding of the correlated bits over independent AWGN channels. The encoded bits are transmitted over the channels by using binary phase-shift keying (BPSK) modulation.

To have an efficient BER performance, two types of optimized degree distributions over AWGN channels with rates 0.5 and 0.32 are used [14]. The corresponding variable node degree distribution for code rate 0.5 is given by [14, Table I] $\lambda_{A}(x)=0.25105 x+0.30938 x^{2}+0.00104 x^{3}+0.43853 x^{9}$,

and for code rate 0.32 is calculated as following

$$
\lambda_{B}(x)=0.3127 x+0.3582 x^{2}+0.04 x^{6}+0.2891 x^{9},
$$

both of which have maximum variable degree 10. A modified progressive edge growth (PEG) method is used to construct relatively short-length parity-check matrices with very low error-floor performance [15].

Just for the simplicity of the iterative decoders, we assume the systematic parity-check matrices with equal rates for the both channels.

\section{B. Theoretical Bound}

Let $H\left(s_{1}, s_{2}\right)$ denote the joint entropy of the first and the second sources. According to the SW bounds, the total required rate to transmit the correlated information sequences over the both channels is given by

$$
R=H\left(s_{1}, s_{2}\right) /\left(1 / R_{c_{1}}+1 / R_{c_{2}}\right),
$$

where $R_{c_{i}}$ for $i \in\{1,2\}$ is the code rate of the $i$-th channel, where the terminologies and/or symbol notations follow [5] and [6]. The energy per source bit, $E_{s o}$, can then be related to the energy per information bit, $E_{b}$, and the energy $E_{s}$ per sent symbol to be sent over the channels is given by

$$
2 E_{s o}=H\left(s_{1}, s_{2}\right) E_{b}=\left(1 / R_{c_{1}}+1 / R_{c_{2}}\right) E_{s},
$$

where $E_{s}=1$ in BPSK modulation.

In general, the parity-check matrices of each channel can be different in code rates. However, we only focus on the cases that both channels use the same rate of the codes (symmetric case), i.e., $R_{c_{1}}=R_{c_{2}}$, hence the Shannon/SW limit for calculation of $E_{s o} / N_{0}$ in the symmetric case is given by

$$
\left.\left(\frac{E_{s o}}{N_{0}}\right)\right|_{l i m}=\frac{2^{H\left(s_{1}, s_{2}\right) R_{c_{1}}}-1}{2 R_{c_{1}}},
$$

where $\sigma_{n}^{2}$ and $N_{0}=2 \sigma_{n}^{2}$ respectively denote the noise variance of the two AWGN channels, which is also assumed to be equal, and the noise power spectral density.

Moreover, $H\left(s_{1}, s_{2}\right)=H\left(s_{1}\right)+h(p)$ where $H\left(s_{1}\right)$ is the entropy rate of the binary Markov source and $h(p)$ is the entropy of the correlation parameter. 


\section{Joint Source-Channel Decoder}

In the class of JSCD, which the proposed technique also belongs to, the processing of correlation between the sources is performed at the decoder side. Hence, the design of the joint decoder that can best exploit the source-channel code properties plays very important role. In the proposed decoder, the temporal and source correlations are exploited in two consecutive iterations, these iterations are referred to as local (horizontal) and global (vertical) iterations. During the horizontal iterations, the systematic variable nodes have LLR inputs from the received signals and a BCJR decoder matched to the trellis diagram of the Markov source. The LLRs are exchanged between variable and check nodes of the LDPC code until either no more relevant improvement in LLR is achieved or a maximum number of local iterations is reached. Then, the correlation between two bit sequences is estimated at the end of the horizontal iterations and the LLR associated to the source correlation is passed on to the systematic variable nodes of each channel via the vertical iteration. For the initialization, the LLRs to be used in the vertical iteration are first set zero for the both decoders.

In Fig. 1 the block diagram of the proposed JSCD structure is presented.

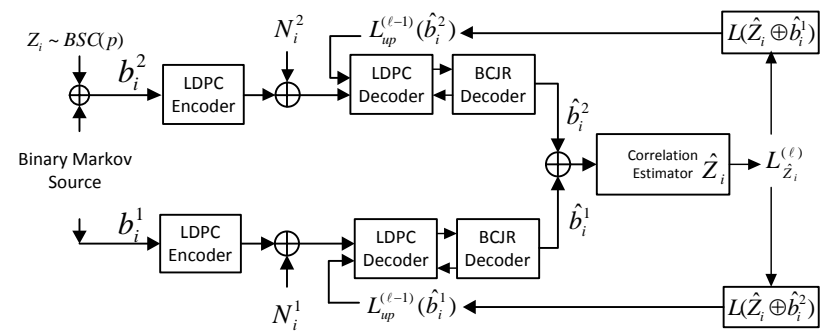

Fig. 1: Block diagram of the proposed JSCD technique for correlated binary sequences generated by a Markov source.

Let us consider the $j$-th local and the $\ell$-th global iteration of the proposed decoder. Let $L_{v, c}^{(j)}$ and $L_{c, v}^{(j)}$ respectively denote the LLR messages passed from a variable node $v$ to a check node $c$ and from the $c$-th check node to the $v$-th variable node at the $j$-th iteration. Also, $L_{v}^{c c}$ represents the LLR obtained from the received signal to be input to the variable node $v \in$ $\{1, \ldots, n\}$. Thus, $L_{v}^{c c}=2 r_{v} / \sigma_{n}^{2}$ where $r_{v}$ is the received noisy signal. The index of the channels is omitted for the simplicity, so far as no ambiguity is expected in the notations.

The process for LLR message updating in the variable nodes follows the standard SP decoding with additional LLRs from the BCJR decoder with a modification due to the source correlation, as detailed for the expressions of Eq. 7 . 9 The information exchange between the SP and the Markov decoders does not contain the information obtained by the previous iterations, and hence is extrinsic information.

The LLR messages to be forwarded from the $k$ systematic variable nodes to the corresponding check nodes are given by

$$
L_{v, c}^{(j)}=L_{v}^{c c}+\sum_{c^{\prime} \neq c} L_{c^{\prime}, v}^{(j-1)}+L_{M, v}^{(j-1)}+L_{v, u p}^{(\ell-1)},
$$

where $v \in\{1, \ldots, k\}, L_{M, v}^{(j)}$ and $L_{v, u p}^{(\ell)}$ denote the extrinsic LLR, sent from the Markov decoder to the variable node $v$ and the LLR already updated according to the correlation, respectively, in the $j$-th and the $\ell$-th iterations. It is worth mentioning that summation is performed only on the check nodes connected to the variable node $v$.

For parity variable nodes, the updated messages are obtained in the same way as the standard SP decoder, and is represented by

$$
L_{v, c}^{(j)}=L_{v}^{c c}+\sum_{c^{\prime} \neq c} L_{c^{\prime}, v}^{(j-1)}
$$

where $v \in\{k+1, \ldots, n\}$. The messages $L_{c, v}^{(j)}$ are also updated in each local iteration exactly in the same way as the standard SP decoder.

The input extrinsic LLR of the Markov decoder is calculated by subtracting the extrinsic LLR of the Markov decoder, obtained as the result of previous Markov decoding, from the a posteriori LLR, obtained as the result of the current SP decoding, in each local iteration. Thus, the sent LLR from a variable node $v$ to the Markov decoder in the $j$-th iteration, denoted as $L_{v, M}^{(j)}$, is calculated as

$$
L_{v, M}^{(j)}=L_{v}^{c c}+\sum_{c^{\prime}} L_{c^{\prime}, v}^{(j-1)}+L_{v, u p}^{(\ell-1)}
$$

where $v \in\{1, \ldots, k\}$. Furthermore, the BCJR decoder which uses the trellis diagram representing the Markov source is applied to calculate the extrinsic $L_{M, v}^{(j)}$ values by the same equations represented in [9].

During the vertical iterations, the LLR $L\left(\hat{z}_{v}\right)$ of the binary error sequence, $z_{v}$, is estimated. The estimate of the binary error, $\hat{z}_{v}^{(\ell)}$, in the $\ell$-th vertical iteration, is given by

$$
\hat{z}_{v}^{(\ell)}=\hat{b}_{v}^{1,(\ell)} \oplus \hat{b}_{v}^{2,(\ell)},
$$

where $\hat{b}_{v}^{q,(\ell)}, q=1,2$, is the estimated source (systematic) bits of the $q$-th channel obtained as the result of the horizontal iterations. Hence, $\hat{b}_{v}^{q,(\ell)}$ is calculated as

$$
\hat{b}_{v}^{q,(\ell)}=\left\{\begin{array}{ll}
0 & \text { if } L_{v}^{q,(\ell)} \geq 0 \\
1 & \text { if } L_{v}^{q,(\ell)}<0
\end{array},\right.
$$

where $L_{v}^{q,(\ell)}=L_{v}^{q, c c}+L_{M, v}^{q,\left(j_{t}-1\right)}+L_{v, u p}^{q,(\ell-1)}+\sum_{c^{\prime}} L_{c^{\prime}, v}^{q,\left(j_{t}-1\right)}$ and $j_{t}$ is the terminated horizontal iteration. The LLR $L\left(\hat{z}_{v}^{(\ell)}\right)$ of $\hat{z}_{v}^{(\ell)}$ is estimated using the method presented in [7], as

$$
L\left(\hat{z}_{v}^{(\ell)}\right)=\left(1-2 \hat{z}_{v}^{(\ell)}\right) \frac{k-W_{H}}{W_{H}},
$$

where $k$ and $W_{H}$ denote the length of the original source packet and Hamming weight of estimated binary error vector $\hat{\mathbf{Z}}^{(\ell)}=\left\{\hat{z}_{1}^{(\ell)}, \ldots, \hat{z}_{k}^{(\ell)}\right\}$.

Finally, according to the modulo- 2 addition of the error sequence, the updating LLRs of the first and the second concatenated decoders are given by

$$
\begin{aligned}
& L_{v, \text { up }}^{1,(\ell)}=2 \operatorname{atanh}\left(\tanh \left(L\left(\hat{z}_{v}^{(\ell)}\right) / 2\right) \tanh \left(L_{v}^{2,(\ell)} / 2\right)\right), \\
& L_{v, u p}^{2,(\ell)}=2 \operatorname{atanh}\left(\tanh \left(L\left(\hat{z}_{v}^{(\ell)}\right) / 2\right) \tanh \left(L_{v}^{1,(\ell)} / 2\right)\right),
\end{aligned}
$$


where $\tanh ($.$) and \operatorname{atanh}($.$) denote, respectively, the hyper-$ bolic and inverse hyperbolic tangent functions.

\section{SIMULATION RESULTS}

In this section, we demonstrate the advantages characteristics of the JSCD technique rather than using a single structure which only exploits either the temporal or source correlations. The distributed source-channel (DSC) coding was performed for symmetric and asymmetric binary Markov sources with different code rates over AWGN channels. In both examples, the maximum number of local and global iterations for the proposed JSCD technique was set at 50 and 15, respectively.

Example 1: In this example, a symmetric binary Markov source with the state transition parameters $\alpha=\beta=0.1$ was considered. For DSC coding of the correlated transmitted bits, two different irregular LDPC codes with the degree distribution $\lambda_{A}$, the rate $R_{c}=0.5$, and the block-length $n=4096$ were constructed by using a modified PEG method [15].

The FER/BER performances of the transmitted source bits with different decodings are presented in Fig. 2, including the proposed JSCD, the concatenated SP-BCJR decoding, the decoding method proposed in [7], and the standard SP decoding. It is found that roughly $1.3 \mathrm{~dB}$ gain in terms of $E_{s o} / N_{0}$ is achieved by the proposed decoder with the correlation parameter $p=0.01$ compared to the case where only the temporal correlation of the source is used. The gap between theoretical limits 1 and our simulation results for the standard SP decoder, the decoder proposed in [7], the concatenated SP-BCJR decoder and the proposed decoder are $1.5 \mathrm{~dB}, 3.5 \mathrm{~dB}, 2.9 \mathrm{~dB}$ and $4 \mathrm{~dB}$, respectively.

The FER/BER curves of the JSCD method with different correlation parameters and their respective Shannon/SW limits are shown in Fig. 3. The gap between the theoretical limits and the simulation results for the JSCD with different correlation parameters $p \in\{0.01,0.05,0.1,0.2\}$ are $\{4,2.9,2.2,1.5\}$ $\mathrm{dB}$, respectively. It is found that the more the correlation is between the sources, the more the gap is between the theoretical limits and the simulation results. The gap indicates that there will remain many open questions for the optimal code design and decoding algorithm development which are left as a future study.

Example 2: The proposed JSCD technique was also performed for an asymmetric binary Markov source with $\alpha=0.1$ and $\beta=0.2$. Two different irregular LDPC codes were constructed by the modified PEG method for encoding of correlated sources by using the degree distribution $\lambda_{B}$ with the rate $R_{c}=0.32$, and the block-length $n=6400$.

The FER/BER performance of the proposed decoder with the correlation parameter $p=0.01$ is demonstrated in Fig. 4 In this figure, the FER/BER results of the SP decoder, the decoder proposed in [7], and the concatenated SP-BCJR decoder are also depicted. As shown in Fig. 4 more than

${ }^{1}$ The theoretical limits are obtained using the same techniques described in [5], [16].

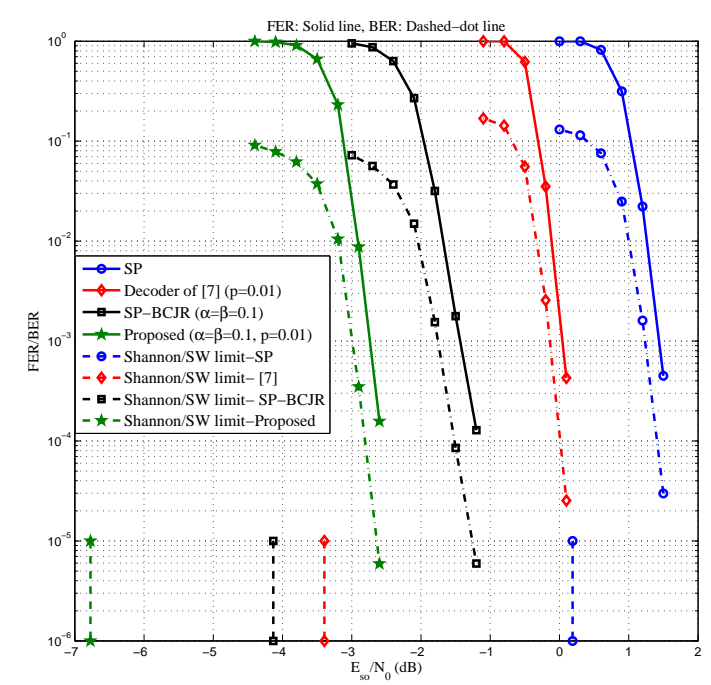

Fig. 2: FER, BER, Shannon/SW limit performances of various decoders for correlated bit sequences with Markov source parameters $\alpha=\beta=0.1$, and $p=0.01$ (Example 1).

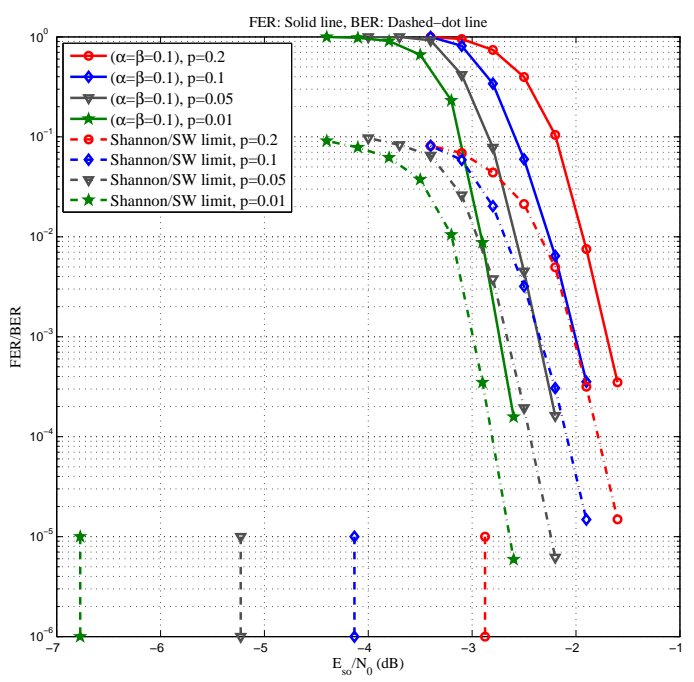

Fig. 3: FER, BER, and Shannon/SW limit performances of the proposed decoder for different correlation values with Markov source parameters $\alpha=\beta=0.1$ (Example 1).

$1.4 \mathrm{~dB}$ gain in terms of $E_{s o} / N_{0}$ is achieved over the decoders that only use either the temporal or source correlations. In this case, the gap between the theoretical limits and the simulation results for the SP decoder, the decoder presented in [7], the concatenated SP-BCJR decoder, and the proposed decoder are $1.5 \mathrm{~dB}, 3.5 \mathrm{~dB}, 2.7 \mathrm{~dB}$ and $3.95 \mathrm{~dB}$, respectively.

Moreover, performances of the proposed decoder for different correlation parameters, $p \in\{0.01,0.05,0.1,0.2\}$, and their corresponding limitations are demonstrated in Fig. 5. The gap between the theoretical limits and the numerical results for 


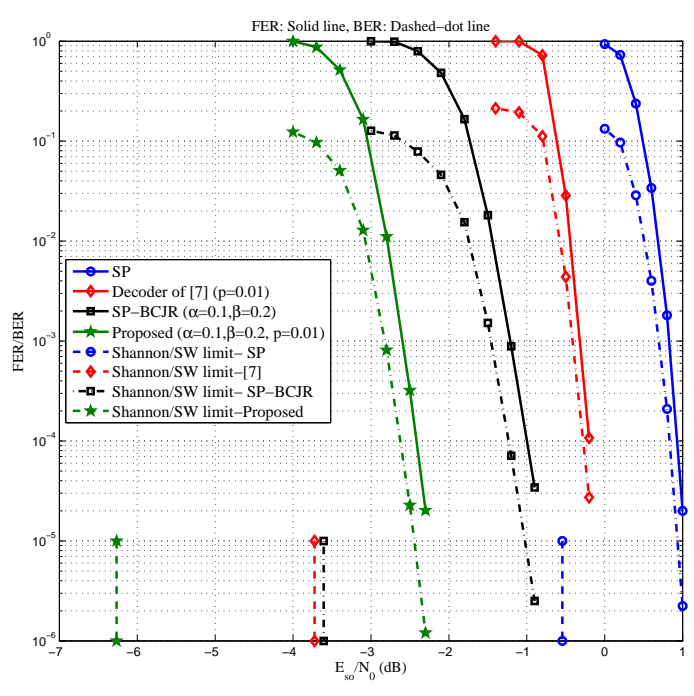

Fig. 4: FER, BER, Shannon/SW limit performances of various decoders for correlated bit sequences with Markov source parameters $\alpha=0.1, \beta=0.2$, and $p=0.01$ (Example 2).

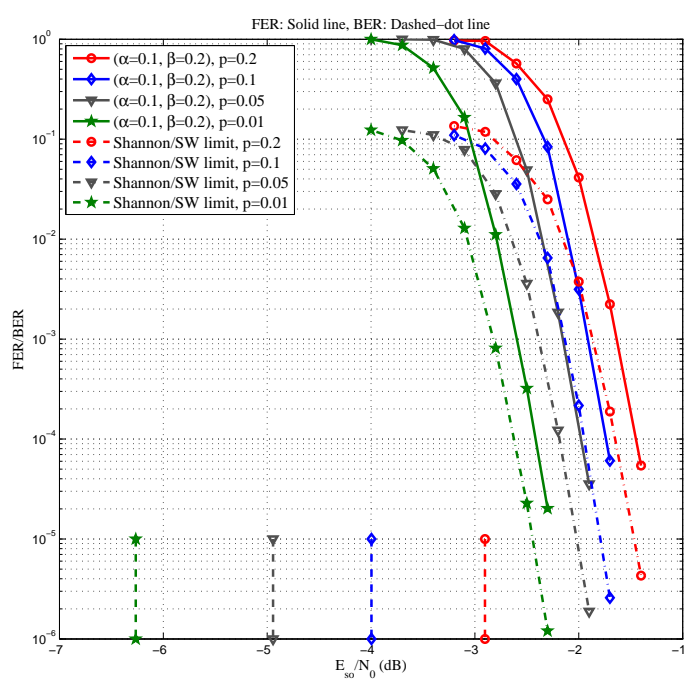

Fig. 5: FER, BER, and Shannon/SW limit performances of the proposed decoder for different correlation values with Markov source parameters $\alpha=0.1, \beta=0.2$ (Example 2).

the correlation parameters described above are, respectively, $\{3.95,2.9,2.2,1.5\} \mathrm{dB}$. It is obvious that the more the correlation is, the more the gap is between the theoretical limits and the simulation results just like the example 1

\section{CONCLUSION}

We have introduced an iterative joint source-channel decoding technique for the transmission of two correlated binary Markov sources. Each of the sources is independently encoded by a relatively short-length irregular LDPC code. The proposed decoder contains two concatenated SP-BCJR decoders, each taking into account the temporal correlation (Markov memory structure) over which extrinsic information is exchanged. Moreover, the output of extrinsic LLRs from the Markov decoders is updated by exploiting the source correlation during the global iterations. The proposed JSCD technique outperforms the other decoders that do not fully utilize the correlation information. Even though the proposed decoding effectively utilizes temporal and source correlations, there still remains a gap between the threshold $E_{s o} / N_{0}$, obtained by the simulations, and the theoretical limits. This indicates that there are many open questions for the optimal code design and decoding algorithm development which are left as a future study.

\section{ACKNOWLEDGMENT}

This work was supported by the Academy of Finland under SWOCNET project.

\section{REFERENCES}

[1] D. Slepian and J. K. Wolf, "Noiseless coding of correlated information sources," IEEE Trans. Inform. Theory, vol. 19, no. 7, pp. 471-480, July 1973.

[2] V. Toto-Zarasoa, A. Roumy, and C. Guillemot, "Non-asymmetric Slepian-Wolf coding of non-uniform Bernoulli sources," Proc. 6th Intern. Symp. Turbo Codes (ISTC), Brest, France, 2010, pp. 314-318.

[3] E. Dupraz, F. Bassi, T. Rodet, and M. Kieffer, "Distributed coding of sources with bursty correlation", Proc. ICASSP 2012, Kyoto, 2012, pp. 2973-2976.

[4] J. Barros and S. D. Servetto, "Network information flow with correlated sources," IEEE Trans. Inform. Theory, vol. 52, no. 1, pp. 155-170, Jan. 2006.

[5] J. Garcia-Frias and Ying Zhao, "Near-Shannon/Slepian-Wolf performance for unknown correlated sources over AWGN channels," IEEE Trans. Commun., vol. 53, no. 4, pp. 555-559, April 2005.

[6] J. Garcia-Frias, Ying Zhao, and W. Zhong, "Turbo-like codes for transmission of correlated sources over noisy channels," IEEE Sig. Process. Mag., vol. 24, no. 5, pp. 58-66, Sept. 2007.

[7] F. Daneshgaran, M. Laddomada, and M. Mondin, "LDPC based channel coding of correlated sources with iteartive joint decoding," IEEE Trans. Commun., vol. 54, no. 4, pp. 577-582, April 2006.

[8] K. Anwar and T. Matsumoto, "Accumulator-assisted distributed turbo codes for relay systems exploiting source-relay correlation," IEEE Commun. Letters, vol. 16, no. 7, pp. 1114-1117, July 2012.

[9] X. Zhou, M. Cheng, K. Anwar, and T. Matsumoto, "Distributed joint source-channel coding for relay systems exploiting spatial and temporal correlations," Proc. Wireless Advanced (WiAd), London, 2012, pp. 7984.

[10] D. MacKay, "Good error-correcting codes based on very sparse matrices," IEEE Trans. Inform. Theory, vol. 45, no. 2, pp. 399-431, March 1999.

[11] L. R. Bahl, J. Cocke, F. Jelinek, and J. Raviv, "Optimal decoding of linear codes for minimizing symbol error rate," IEEE Trans. Inform. Theory, vol. 20, no. 3, pp. 284-287, March 1974.

[12] T. M. Cover and J. A. Thomas, Elements of information theory 2nd Edition. USA: John Wiley \& Sons Inc., 2006.

[13] V. Toto-Zarasoa, A. Roumy, and C. Guillemot, "Hidden Markov model for distributed video coding," Proc. IEEE Intern. Conf. on Image Proc. (ICIP), Hong-Kong, 2010, pp. 3709-3712.

[14] T. J. Richardson, M. A. Shokrollahi, and R. Urbanke, "Design of capacity approaching irregular low-density parity-check codes," IEEE Trans. Inform. Theory, vol. 47, no. 2, pp. 619-637, Feb. 2001.

[15] S. Khazraie, R. Asvadi, and A. H. Banihashemi, "A PEG construction of finite-length LDPC codes with low error floor, "IEEE Commun. Letters, vol. 16, no. 8, pp. 1288-1291, Aug. 2012.

[16] G.C. Zhu and F. Alajaji, "Joint source-channel turbo coding for binary Markov sources," IEEE Trans. Wireless Commun., vol. 5, no. 5, pp 1065-1075, May 2006 